\title{
MAC Anesthesia during Endoscopic Percutaneous Gastrostomy (PEG) in a Patient with Amyotrophic Lateral Sclerosis (ALS). A Case Report
}

\author{
Majlinda NAÇO ${ }^{1}$, Haxhire Gani ${ }^{1}$, Arvin Dibra ${ }^{2}$, Eden Naço ${ }^{3}$, Suzana \\ Mukaj4. \\ https://doi.org/10.32391/ajtes.v3i2.61
}

\section{Abstract}

Background: Amyotrophic lateral sclerosis (ALS) is a serious disease in which some brain and spinal cells die slowly. These nerve cells are called motor neurons and control the muscles that allow the body parts to move. Initially, the disease causes bodily fatigue and muscular weakness through degeneration of the upper and lower motor neurons, leading to loss of ability to initiate and control the voluntary bodily functions. In late stages this disease causes difficulty in the normal swelling and eating that causes the need for PEG in patients with ALS. This clinical picture increases the difficulty and the anesthetic risk used during the PEG application.

Case presentation: We are describing our experience in a 56-year-old male patient, ASA III suffering from ALS and being admitted to our clinic to insert PEG. The patient presented $\mathrm{BMI}=15.6$. After the patient provide informed consent, we were monitoring him for detect changes in pulse oximetry, noninvasive blood pressure, heart rate and visual assessment of ventilator activity, level of consciousness and discomfort. We used balanced propofol for moderate sedation (BPS), loading dose $1 \mathrm{mg}$ midazolam, $0.1 \mathrm{mg}$ of fentanyl, and 5-15 mg propofol in smaller bolus dose were applied for more precise dose titration. The operation was performed after the patient lost the consciousness and had no pupil reflex. The EGD entered to the stomach. Fistula was localized after the visualization of light from the tube. Local lidocaine was applied and $1 \mathrm{~cm}$ incision on the abdominal wall gave the possibility for application of fistula and gastro stoma placement. The patient wakes up 10 minutes after the end of the procedure without using antidotes. The patient after treatment went home in two days using gastro stoma for enteral feeding.

Keywords: Amyotrophic lateral sclerosis, MAC, PEG, BMI
\end{abstract}

Corresponding Author: Majlinda Naço

$\equiv$ = E-mail: majlinda_naco@yahoo.com

${ }^{1}$ The Service of Anesthesia and Intensive Care in General Surgery, HUC "Mother Theresa",

Tirana, Albania.

2 The General Surgery Service of HUC "Mother Theresa", Tirana, Albania.

"Medical student in the catholic university "Our lady of good council", Tirana, Albania.

${ }^{4}$ The Service surgical nurse in General Surgery, HUC "Mother Theresa", Tirana, Albania. 


\section{Full Text}

\section{Introduction}

Amyotrophic lateral sclerosis (ALS) is a group of rare neurological diseases, that is known with different names as Motor Neurone Disease (MND), Charcot's disease, Lou Gehrig's disease is a neurodegenerative disease. It characterized by progressive muscular paralysis reflecting degeneration of motor neurons in the primary motor cortex, corticospinal tracts, brainstem and spinal cord. (1) Amyotrophic lateral sclerosis (ALS) that usually affected the nerve cells (neurons) serve for controlling voluntary muscle movement. Voluntary muscles produce movements like chewing, walking, and talking. The disease is progressive, meaning the symptoms get worse over time. Nowadays there is no cure for ALS but treatment can relieve symptoms and raise the quality of life. The incidence of sporadic amyotrophic lateral sclerosis (SALS) in the 1990's is reported to be between 1.5 and 2.7 per 100,000 population/year (average 1.89 per $100,000 /$ year) in Europe and North America (2). Dysphagia is a common symptom of ALS and leads to increased risk of aspiration, malnutrition, weight loss and dehydration. Mostly patients with ALS live 3-5 years after diagnosis but only $10 \%$ live more than 10 years after first symptoms. Malnutrition and dehydration can also occur inpatients whom have severe upper limb weakness, especially if they live alone for social reason, as this leads to difficulties in meal preparation and consume or prolonged meal times. ALS is associated with a hyper metabolic state; therefore, patients require increased calorie intake $(3,4)$. Most guidelines state that supplementary enteral feeding should be considered when body weight falls by $>10 \%$ of the pre-diagnostic or baseline weight $(5,6)$. The three options available for enteric feeding include percutaneous endoscopic gastrostomy (PEG), percutaneous radiologic gastrostomy (PRG) or radiologically inserted gastrostomy (RIG), and nasogastric tube (NGT) feeding. PEG is the standard procedure for enteral feeding, although the procedure requires mild sedation and therefore has implications in patients with respiratory weakness. To minimize risks, evidence suggests that PEG should be performed before VC falls below $50 \%$ of predicted $(5,7)$ A percutaneous endoscopic gastrostomy seemed a better, though potentially hazardous, alternative in view of the often severely restricted pulmonary function of these patients. Therefore, we prospectively investigated the use of percutaneous endoscopic gastrostomy in our patients with amyotrophic lateral sclerosis. This clinical picture increases the difficulty and the anesthetic risk used during the PEG application therefore we decided to used monitor anesthesia care (MAC) for safety of patient.

\section{Case presentation}

A 56-year-old male patient, ASA III suffering from ALS and with swallowing difficulties admitted for elective operative treatment to the first clinic of general surgery to insert PEG. 
The patient presented $\mathrm{BMI}=15.6$ and he had a period of 4 years diagnosis. The patient was with mild pulmonary dysfunction After the patient provide informed consent, we was monitoring him for detect changes in pulse oximetry, non-invasive blood pressure, heart rate and visual assessment of ventilator activity, level of consciousness and discomfort. We used balanced propofol for moderate sedation (BPS), loading dose $1 \mathrm{mg}$ midazolam, $0.1 \mathrm{mg}$ of fentanyl, and 5-15 $\mathrm{mg}$ propofol in smaller bolus dose were applied for more precise dose titration. The operation was performed after the patient lost the consciousness and had no pupil reflex. The EGD entered to the stomach. Fistula was localized after the visualization of light from the tube. Local lidocaine was applied and $1 \mathrm{~cm}$ incision on the abdominal wall gave the possibility for application of fistula and gastrostoma placement. The patient wakes up 10 minutes after the end of the procedure without using antidotes. the vital parameters were normal with used 5 liters of $\mathrm{O} 2$ nasal. The patient after 2 days intensive care treatment will total parenteral nutrition went home using gastrostoma for enteral feeding after 48 hours.

\section{Discussion}

The management of ALS/MND has considerably changed over the past two decades, with an emphasis on coordinated multidisciplinary care between specialist, community-based therapists and palliative care teams. Although the condition is considered incurable, many of the symptoms arising during the course of the disease are treatable, and all efforts should be made to improve quality of life and help maintain the patient's autonomy for as long as possible. Patients with ALS and their relatives are likely to suffer from depression, feelings of hopelessness and anxiety regarding end-of-life issues following the diagnosis or as the disease progresses (9). Symptomatic treatments aim to improve quality of life of patients and care givers. Malnutrition, specifically under-nutrition which is typically associated with disease. PEG is the modality of choice for long-term enteral access for patients who are unable to maintain sufficient oral intake. Contraindications to PEG placement include pharyngeal or esophageal obstruction, major coagulopathy, abdominal wall abnormalities and the presence of prior abdominal surgery involving stomach and splenic flexure of the colon. Although generally safe, PEG placement can be associated with many potential complications like pneumoperitoneum, entero-cutaneous fistula, small bowel or colon injury, splenic or hepatic injury, intra or retroperitoneal bleeding and abdominal wall bleeding (10). Swallowing difficulties and diaphragmatic weakness are usually late features (11). Respiratory insufficiency occurs commonly in patients with ALS and is a major cause of mortality. PEG is the standard procedure for enteral feeding, although the procedure requires mild sedation and therefore has implications in patients with respiratory weakness. (59). The team of anesthesiologists may be asked to provide anesthesia for procedure that require sedation, monitor anesthesia care (MAC) or 
general anesthesia. MAC is a type of anesthesia service during which a patient is typically still aware, but very relaxed. During endoscopy the patients are in a minimal, moderate or deep "procedural sedation" which allows the specialist to perform the procedure and aims patient's safety and comfort. The common medicines used for sedation are benzodiazepines as midazolam which act on the brain and SNQ and opioids as fentanyl which decrease the patient's perception of pain, dexmedetomidine, low dose ketamine or propofol. Propofol is ideally suited for endoscopy sedation because it is a short acting anesthetic induction drug but always is required greater level of monitoring (11).

\section{Conclusions}

Conscious sedation MAC may be considered safe because less drugs are typically administered during PEG insertion in ALS patients and these did not lead to respiratory complications or to an increase the other complications. Our data indicate that conscious sedation can be used safely in ALS patients with mild to moderate pulmonary dysfunction.(12) However, the application of sedatives and analgesics should be titrated carefully after monitoring the patient's level of consciousness and hemodynamic value to avoid central respiratory depression and airway obstruction, since the airway of the patient is not secured during MAC. It should be chosen as a proper anesthetic alternative after patient comorbidities and preferences in all the type of endoscopic procedures. The choice of sedative and analgesic is based on the required depth of sedation and analgesia of each procedure. The presence of a sufficiently experienced anesthesiologist, as well as oxygen supply, monitoring devices and emergency equipment, are required during MAC both in and outside the operating room.

\section{References}

1. Lokesh C Wijesekera ${ }^{\# 1}$ and P Nigel Leigh $\otimes^{\# 1}$ Amyotrophic lateral sclerosis Orphanet J Rare Dis. 2009; 4: 3.Published online 2009 Feb 3. doi: 10.1186/17501172-4-3.

2. Worms PM. The epidemiology of motor neuron diseases: a review of recent studies. J Neurol Sci. 2001;191:39. [PubMed] [Google Scholar]

3. Desport JC, Preux PM, Magy L, Boirie Y, Vallat JM, Beaufrere B, Couratier P. Factors correlated with hypermetabolism in patients with amyotrophic lateral sclerosis. Am J Clin Nutr. 2001;74:328334. [PubMed] [Google Scholar]

4. Kasarskis EJ, Berryman S, Vanderleest JG, Schneider AR, McClain CJ. Nutritional status of patients with amyotrophic lateral sclerosis: relation to the proximity of death. Am J Clin Nutr. 1996;63:130137. [PubMed] [Google Scholar]

5. Leigh PN, Abrahams S, AlChalabi A, Ampong MA, Goldstein LH, Johnson J, Lyall R, Moxham J, Mustfa N, Rio A, et al. The management of motor neurone disease. J Neurol 
Neurosurg

Psychiatry. 2003;74:iv32iv47. [PMC free article] [PubMed] [Google Scholar]

6. Andersen PM, Borasio GD, Dengler $R$, Hardiman $O$, Kollewe K, Leigh PN, Pradat PF, Silani V, Tomik B. EFNS task force on management of amyotrophic lateral sclerosis: guidelines for diagnosing and clinical care of patients and relatives. Eur

Neurol. 2005;12:921938. [PubMed] [Google Scholar]

7. Mathus-Vliegen LM, Louwerse LS, Merkus MP, Tytgat GN, Vianney de Jong JM. Percutaneous endoscopic gastrostomy in patients with amyotrophic lateral sclerosis and impaired pulmonary function. Gastrointest Endosc. 1994;40:463469. [PubMed] [Google Scholar]

8. Hu MT, Ellis CM, Al-Chalabi A, Leigh PN, Shaw CE. Flail arm syndrome: a distinctive variant of amyotrophic lateral sclerosis. J Neurol Neurosurg Psychiatry. 1998;65:950951. [PMC free article][PubMed] [Google Scholar]

9. Averill AJ, Kasarskis EJ, Segerstrom SC. Psychological health in patients with amyotrophic lateral sclerosis. Amyotrophic Lateral Sclerosis. 2007;8:243-

254. [PubMed] [Google Scholar] 10. Schrag, S.P., Sharma, R., Jaik, N.P., et al. (2007) Complications related to percutaneous endoscopic gastrostomy (PEG) tubes. A comprehensive clinical review. Journal of Gastrointestinal and Liver Diseases, 16, 407-418. [Citation Time(s):1]

11. Naco M, Gani H,\& BP:Anesthesia in endoscopic procedutes" International Journal of Sciences\& Research www.ijsr.net Online ISSN:23197064. Scientific Journal, research Gate Impact Factor(2018):0.28, SJIF (2018):7.426: Paper ID;ART20198205,Vol.8, Issue 5, Maj 2019..fq 1723-1735.

12. Strijbos D, Hofstede J, Keszthelyi D, Masclee AAM, Gilissen LPL. Percutaneous endoscopic gastrostomy under conscious sedation in patients with amyotrophic lateral sclerosis is safe: an observational study.Eur J Gastroenterol Hepatol. 2017 Nov;29(11):1303-1308. doi: 10.1097/MEG.0000000000000959 .PMID:28877087 